\title{
Avaliação da Aplicação da Aprendizagem Baseada em Problemas na Disciplina de Interação Humano e Computador de curso de Engenharia de Software
}

\begin{abstract}
André Barros de Sales - Universidade de Brasília - UnB - andrebdes@unb.br Arthur de Moura Del - Universidade de Brasília - UnB - arthurmde@gmail.com Márcia B.de Sales - Universidade Federal de Santa Catarina - marcia.barros@ufsc.br

Resumo: A busca por abordagens didáticas para melhorar o processo de ensinoaprendizagem tem sido uma instigação para alguns educadores. Nesse contexto, e diante dessa necessidade, tem-se experimentado a metodologia de Aprendizagem Baseada em Problemas (PBL - Problem Based Learning) em disciplinas de cursos de graduação. Este artigo apresenta o uso da PBL na disciplina de Interação Humano-Computador do curso de Engenharia de Software da Faculdade UnB Gama da Universidade de Brasília. A pesquisa envolveu a participação de 28 alunos, que analisaram e avaliaram essa metodologia por meio de questionários durante três semestres consecutivos. Observouse que os alunos demonstraram satisfação com a utilização dessa metodologia empregada nas aulas dessa disciplina.
\end{abstract}

Palavras-chave: Interação humano-computador. Aprendizagem baseada em problemas. Abordagem didática.

\section{Evaluating the Implementation of Problem Based Learning Approach in Human-Computer Interaction Discipline from Software Engineering Course}

\begin{abstract}
The search for didactic approaches to improve the process of teaching and learning has been an instigation to some educators. In this context and in the face of this need, the methodology of Problem Based Learning (PBL - Problem Based Learning) has been experienced in the disciplines of undergraduate courses. This paper presents the use of PBL in the Software Engineering course's, from Faculdade UnB Gama from UnB, discipline of Human-Computer Interaction. This research involved the participation of 28 students, which examined and evaluated this methodology by means of questionnaires for three consecutive semesters. It was observed that the students were satisfied with the use of this methodology in the classroom for this course.
\end{abstract}

Key-words: Human-computer interaction. Problem based learning. Didactic approach.

\section{Introdução}

Os cursos oferecidos pela Universidade de Brasília no campus da Faculdade UnB Gama - FGA são Engenharia Automotiva, Engenharia de Software, Engenharia Eletrônica, Engenharia de Energia e Engenharia Aeroespacial. O principal objetivo da presença destes cinco cursos no campus FGA é integrar os conhecimentos dos cursos oferecidos para que os alunos possam, juntos, desenvolver inovações tecnológicas e soluções criativas para problemas comuns. Para tanto, existem projetos de pesquisa e extensão que oferecem a oportunidade para que os alunos do campus utilizem seus conhecimentos, trabalhando em grupos e orientados por um ou mais professores. Os 
alunos que participam desses projetos também desenvolvem características muito importantes, como trabalho em grupo, liderança e aplicação de conhecimentos distintos num mesmo contexto, entre outros. Tais características são fundamentais para a atuação do profissional no mercado de trabalho, podendo ser utilizadas nos mais variados contextos.

Algumas disciplinas específicas dos cursos da FGA também trabalham as características citadas acima em seus diferentes conteúdos e competências, como a IHC (Interação Humano Computador), disciplina ministrada no quinto semestre de Engenharia de Software. A IHC trabalha conceitos de design de interface, cognição humana e princípios de usabilidade que são aplicados no desenvolvimento de um software ou de qualquer aplicativo que exija interação de um usuário com o computador. Todavia, o conteúdo abordado na disciplina pode e deve ser utilizado em outros contextos sociais, por exemplo. A capacidade de criação de design de software pode ser utilizada no ramo de propaganda e marketing, os conceitos de cognição humana podem ser explorados no desenvolvimento de materiais mais adequados a idosos e os princípios de usabilidade podem ser aplicados nos projetos físicos (por exemplo: projeto de ônibus mais confortável) ou para projetos digitais (desenvolvimento de: sistemas, sites, vídeo-aulas etc).

Considerando a interdisciplinaridade existente em IHC (ergonomia, psicologia, ciência da computação, artes, design, semiótica, linguística e áreas afins), o conteúdo abordado na disciplina e sua aplicação, surge a necessidade de oferecer ao aluno recursos didáticos que o envolva no processo de aprendizagem como (Sales, Coelho e Sales, 2012) e os resultados obtidos no estudos por (Júnior, 2010; Cerutti, 2010) foi adotada na disciplina IHC, no segundo semestre de 2011, a metodologia de aprendizagem baseada em problemas (PBL) que é uma estratégia pedagógica centrada no aluno.

Este artigo relata a abordagem pedagógica adotada na disciplina IHC, no segundo semestre de 2011. Como já se disse acima, a metodologia seguiu a Aprendizagem Baseada em Problema (PBL - Problem Based Learning), estratégia pedagógica centrada no aluno. Cerutti (2010) enfatiza a motivação de explorar e discutir novas práticas pedagógicas e experiências de sala de aula para fornecer subsídios metodológicos aos professores com o intuito de promover a aprendizagem efetiva em IHC.

Tendo em vista a motivação destacada por Cerutti (2010), este artigo apresenta a avaliação dos alunos sobre a metodologia PBL na disciplina Interação HumanoComputador para o curso de Engenharia de Software, relatando a experiência durante três semestres letivos: o segundo de 2011 e os dois semestres de 2012. Na sua primeira seção, este artigo apresenta alguns referenciais teóricos sobre PBL. Na segunda seção, os passos metodológicos da introdução do PBL na IHC e a forma de avaliar tal abordagem. Posteriormente, apresentam-se os resultados obtidos e algumas discussões preliminares. Por fim, na última seção, são tecidas algumas considerações.

Nas seções que se seguem descrevemos os formatos a usar nos títulos, subtítulos e no texto principal do documento, bem como o formato para as legendas de tabelas e figuras e as normas para as referências.

\section{Aprendizagem Baseada em Problemas (PBL)}

A metodologia PBL de ensino e aprendizagem foi introduzida inicialmente na Universidade de McMaster, Canadá, que a implantou na Faculdade de Ciências Médicas em 1969. Entretanto, esse modelo baseado em estudos de caso tem como referencial 
teórico a Universidade de Harvard no século XIX. O PBL tem sido explorado mais recentemente, fato evidenciado pelo crescente número de publicações e relatos de experiências sobre a utilização do método nos mais diferentes ramos e graus de ensino e educação.

Ribeiro et al. (2004) afirmam que a Aprendizagem Baseada em Projetos se caracteriza pelo uso de problemas do mundo real para encorajar os alunos a desenvolverem pensamento crítico e habilidades de solução de problemas, adquirindo conhecimento sobre conceitos essenciais da área estudada. Esses autores ainda evidenciam a utilização do método ao longo de um curso completo. Convém salientar que o método tem sido usado com sucesso como parte integrante da estratégia educacional de determinado curso, não sendo empregado como metodologia principal.

A abordagem do PBL permite que os educadores ministrem aulas aproximando o aluno da realidade das empresas, possibilitando melhor preparo do futuro profissional. Os defensores do PBL (Linge \& Parsons, 2006; Ribeiro et al., 2004; Boscarioli, 2011; Greening et al., 1997) afirmam que o método incentiva o aluno a buscar novos conhecimentos e aprofundar determinados assuntos, aprendendo a fazer, atuar, agir em diferentes contextos, respeitando as diversidades humanas para realizar trabalhos em grupo, desenvolvendo sua maturidade, sensibilidade e responsabilidade. Segundo Rodrigues \& Araújo (2006), um dos fundamentos principais do método é ensinar o aluno a aprender, buscando conhecimento em meios variados. Para isso, diferentemente da abordagem tradicional de ensino, o professor assume papel de tutor e tem responsabilidade de orientar e facilitar o processo de desenvolvimento do projeto, atuando como referência para os alunos.

O potencial da metodologia PBL na disciplina IHC foi explorado por Nordahl \& Serafin (2008). Os autores exploram a capacidade do método para promover a interdisciplinaridade e a transdisciplinaridade (termo usado para definir o maior grau de estudo integrado), nas quais os alunos vão além das disciplinas, iniciando na solução de um problema e trazendo todo o aprendizado dessas disciplinas que contribuam para a solução. Em outro relato de experiência de utilização do PBL em IHC, Boscarioli (2011) afirma que o modelo tradicional de ensino valoriza sobremaneira o conteúdo e a utilização da memória por parte dos alunos, enquanto PBL visa a estimular o desenvolvimento de suas capacidades de raciocínio lógico e de reflexão.

\section{Metodologias - Proposta da Aplicação do PBL em IHC}

Componente das disciplinas obrigatórias do curso de Engenharia de Software da FGA, a IHC admite uma turma de apenas cerca de quinze alunos e é ministrada e coordenada por um docente especializado nos seus conteúdos. A disciplina possui uma sequência de conteúdos programáticos que devem ser apresentados aos alunos no decorrer do semestre letivo, cabendo ao docente escolher a metodologia de ensino ou estratégia didático-pedagógica a ser adotada. De forma geral, o conteúdo a ser explorado envolve métodos, ferramentas e técnicas para o processo e projeto de Iteração Humano-Computador, aspectos de usabilidade e ergonômicos, tipos de iteração, avaliação de interfaces e padrões, entre outros mais específicos.

Tendo em vista o crescimento da utilização da metodologia PBL em diferentes ramos da educação, inclusive na educação de engenharias e em disciplinas semelhantes à IHC, além de relatos de experiências da adoção da metodologia integral ou parcialmente em cursos superiores, principalmente no aprofundamento do conhecimento sobre PBL com base na literatura, essa metodologia foi adotada na 
disciplina IHC do campus FGA nos semestres 02/2011, 01/2012 e 02/2012. O principal objetivo é incentivar o estudo e aguçar o interesse dos alunos pelos conteúdos da disciplina, buscando melhorar a aprendizagem para que os conteúdos ministrados possam ser utilizados nos mais diferentes contextos e na solução de problemas referentes especificadamente ao curso de Engenharia de Software e também atendendo aos objetivos do campus FGA, como descrito no início deste texto.

Com a adoção do PBL, buscou-se desenvolver o conhecimento produzido em IHC, e, tendo em vista os aspectos mercadológicos, explorando-se algumas das três classificações do conhecimento de IHC, como destaca Júnior (2010):

- Variabilidade: retrata a possibilidade de alterar o contexto para resolver problemas, replicando e adaptando o conhecimento adquirido em ambientes distintos do ambiente de aprendizagem;

- Codificação: é a possibilidade de o aluno conseguir explicar, documentar e repassar o conhecimento; e

- Difusão: relacionado à difusão de determinados conhecimentos. O conhecimento difundido é extensível ao maior número de situações, enquanto o não difundido é importante para resoluções inovadoras, consolidação de pesquisas e exploração de novas fronteiras.

Com base em relato de experiência da adoção do PBL em IHC, Boscarioli (2011) observa a necessidade de cuidadoso planejamento e administração de todo o processo pelo professosr, tendo em vista algumas dificuldades referentes ao tempo de contato e interação entre professor e alunos. Assim sendo, buscou-se adequar o PBL da melhor maneira às aulas durante a semana e estruturar os problemas que seriam utilizados na metodologia. Tais problemas foram apresentados aos grupos de alunos (grupos tutoriais) que, na dinâmica de desenvolvimento do PBL, analisaram o problema, formularam os principais objetivos tanto de aprendizagem quanto específicos à resolução, realizaram buscas de informações externamente e sintetizaram e testaram essas informações adquiridas, etapas também destacadas por Pereira et al. (2007).

Ao longo do semestre, foram passados alguns problemas para cada grupo, não simultaneamente, com determinado tempo para formular a solução e avaliar o aprendizado. Esses problemas envolviam situações reais, nas quais soluções, sustentadas por base teórica, eram estudadas e formuladas sob orientação e tutoria do professor.

\subsection{Metodologia de Avaliação do PBL em IHC}

Uma das principais motivações do presente estudo é avaliar e concluir sobre a utilização da metodologia PBL na disciplina IHC. Para tanto, foi desenvolvido um questionário que contém perguntas dissertativas e fechadas, com intuito de avaliar a metodologia PBL e suas características, a viabilidade de sua utilização na disciplina IHC e a maneira como o método foi adaptado à disciplina. Ao fim do semestre letivo esse questionário foi passado para os alunos, que o responderam sem identificar-se.

A primeira parte do questionário é composta por perguntas referentes à utilização do PBL em IHC. Assim, os alunos avaliaram a utilização do PBL na disciplina, os problemas utilizados, práticas, dinâmicas e o desempenho dos alunos nessas atividades. A última questão referente a esse quesito do questionário é sobre as formas avaliativas adotadas para as atividades. Esta parte é composta por seis perguntas, cujas opções de resposta são: Muito Bom, Bom, Regular, Ruim, Muito Ruim.

Para avaliar as características que compõem o PBL adotou-se o mesmo sistema utilizado por Rodrigues \& Araújo (2006). Assim, os alunos avaliaram e elencaram sete 
características do PBL em níveis de importância, devendo ordenar as sete opções que vão de 1 (mais importante) a 7 (menos importante). Estas opções são:

- O método é dinâmico;

- O método possibilita desenvolver competências de solução de problemas;

- O método estimula a busca do conhecimento por meio da pesquisa;

- A pesquisa proporciona a autoaprendizagem;

- O método proporciona a oportunidade de trabalhar em grupo;

- O método origina problemas de relacionamento no grupo; e

- O método instiga o desempenho de papéis dentro do grupo.

Por último, no questionário foram solicitadas as opiniões dos alunos sobre a disciplina, podendo sugerir melhorias e apresentar críticas ao método.

\section{Resultados e Discussões}

Esta seção apresenta os resultados obtidos com base na resposta dos alunos ao questionário aplicado em IHC sobre PBL. O questionário foi respondido por 28 alunos ao longo de três semestre de aplicação da metodologia em IHC. Com o objetivo de melhor apresentar os resultados obtidos na aplicação do questionário, são apresentados gráficos para cada pergunta feita, assim como a discussão dos resultados apresentados. Para manusear as respostas mais objetivamente, elas serão discutidas através de duas variações. Assim, tem-se a resposta A que representa a satisfação e aprovação do aluno quanto ao item explorado, englobando as respostas muito bom e bom. Por outro lado, a resposta B representa a insatisfação do aluno quanto ao item explorado, englobando as respostas regular, ruim e muito ruim. A Figura 1 apresenta os resultados obtidos na avaliação dos alunos quanto à utilização do PBL para IHC.

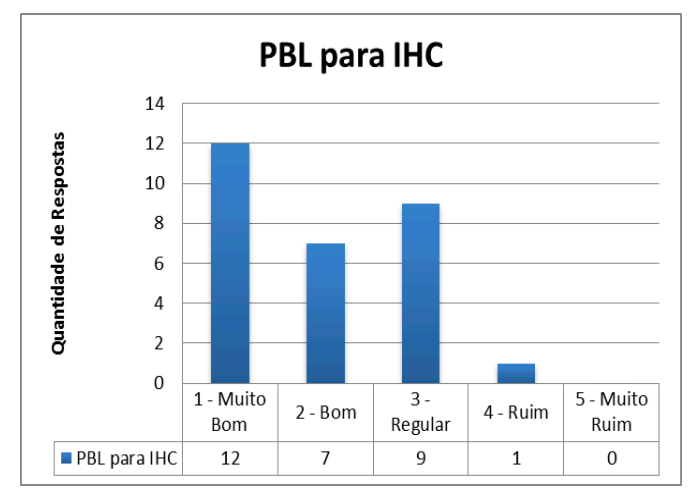

Figura 1 - Avaliação geral da utilização do PBL em IHC

Utilizando-se a estratégia descrita anteriormente, verifica-se que mais de $65 \%$ das avaliações foram positivas quanto à utilização do método PBL em IHC. Este resultado representa, em termos gerais, o grau de aceitação do PBL pelos alunos durante os três semestres citados. A próxima figura apresenta os resultados que avaliam a qualidade dos problemas escolhidos e desenvolvidos durante a disciplina de IHC sob o ponto de vista dos alunos da disciplina. 


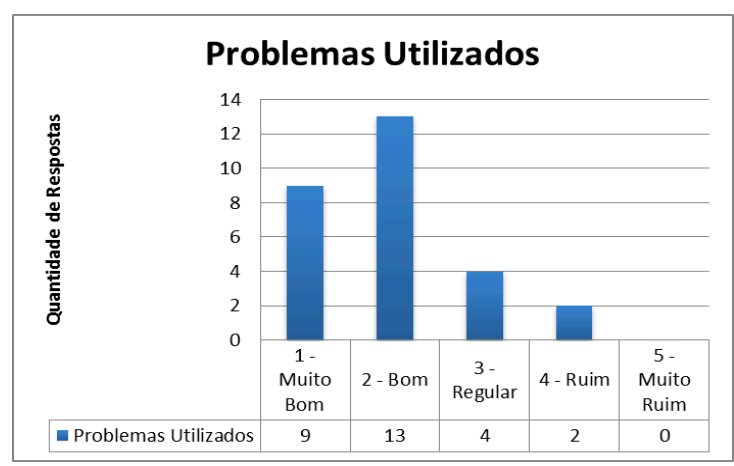

Figura 2 - Avaliação dos problemas utilizados para aplicação do PBL em IHC

Os resultados apresentados na Figura 2 são muito importantes para avaliar qualquer aplicação do PBL, uma vez que os problemas utilizados se tornam os principais meios de aprendizado dos alunos. A partir da visão binária estabelecida, pode-se observar 22 respostas positivas contra apenas seis negativas. Estes resultados devem ser levados em consideração na análise das outras respostas do questionário que avaliam outros aspectos mais específicos, uma vez que todos eles podem sem influenciados pela escolha do problema para aplicar o PBL. Os resultados da próxima figura representam uma autoavaliação dos alunos quanto ao seu esforço individual e desempenho para resolver os problemas durante todo o semestre letivo na disciplina IHC.

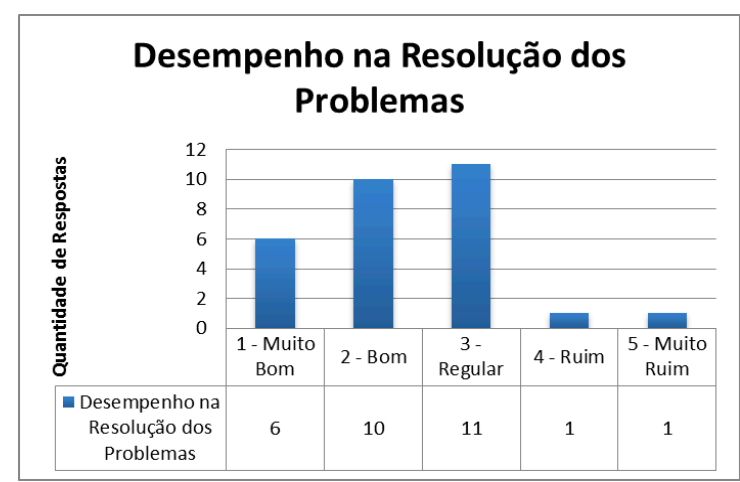

Figura 3 - Autoavaliação dos alunos quanto ao seu desempenho em IHC com PBL

A Figura 3 destaca que 55\% dos alunos avaliam bem seu próprio desempenho durante a realização das atividades de resolução dos problemas de IHC no semestre em que cursou a disciplina. Contudo, em autoavaliação, outros 13 alunos não renderam o esperado ou não avaliaram bem seu desempenho nas atividades relacionadas aos problemas escolhidos. Os resultados negativos podem estar relacionados a aspectos motivacionais intrínsecos ao próprio conteúdo da disciplina, ao trabalho em equipe, ao problema e às características do método PBL, também avaliadas nesse questionário. Outras relações motivacionais podem ser estabelecidas com os tipos de conhecimento destacados neste texto anteriormente. Onde o aluno consegue ver a variabilidade de seu conhecimento na aplicação de vários contextos diferentes, há motivação. Por outro lado, as formas de difusão do conhecimento adquirido, até mesmo para avaliação necessária dos professores, podem inibir o aluno tímido, resultando em queda de motivação. Cumpre lembrar aqui a necessidade de adaptar o método PBL para que alunos possam trabalhar suas habilidades de comunicação e apresentação, características estas bem avaliadas, tanto no âmbito acadêmico quanto no mercado de trabalho.

Outro aspecto inerente à experiência do aluno com o método PBL em IHC foi avaliada na opinião dos alunos sobre a prática requerida na disciplina para utilizar o 
método. Tal questão está diretamente relacionada com os resultados mostrados na Figura 3, que, como já discutido, envolve aspectos motivacionais. $75 \%$ dos alunos avaliaram positivamente a característica do método de requerer muita atividade prática, enquanto os demais optaram pelas respostas regular ou ruim. A seguir, dando continuidade à avaliação de características específicas do PBL, a Figura 4 mostra a avaliação de sete características do PBL.

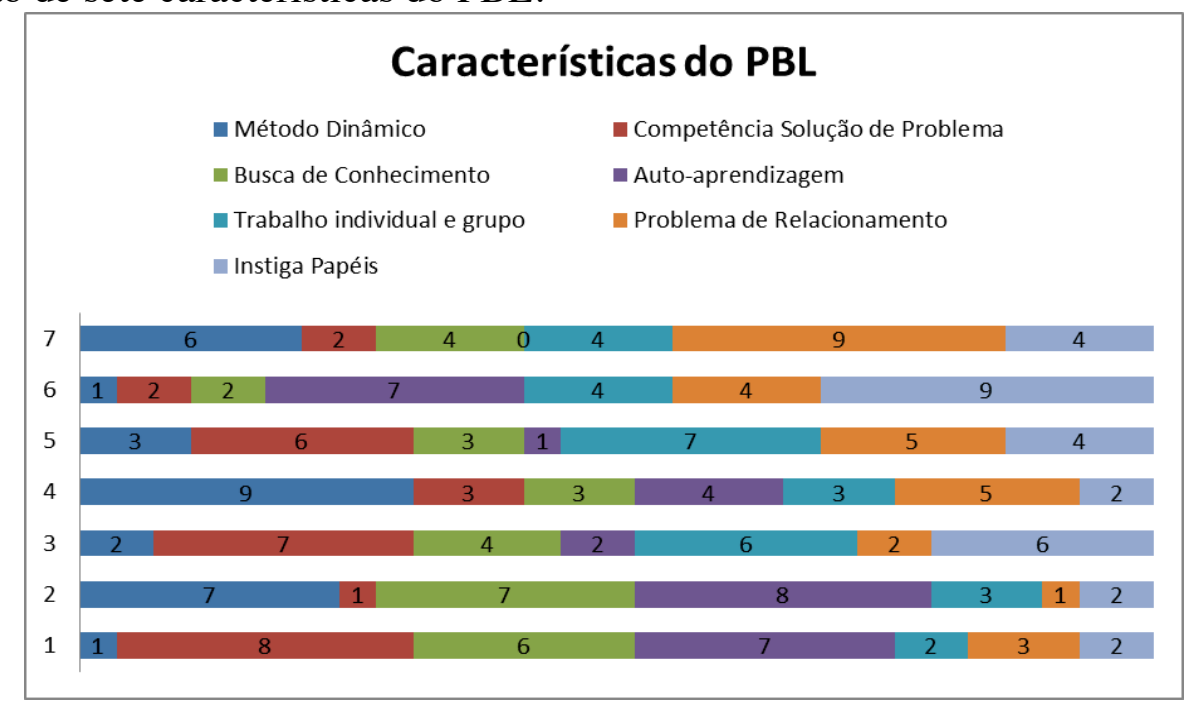

Figura 4 - Avaliação e hierarquização de sete características do PBL.

Esta parte do questionário tinha como principal objetivo verificar, na avaliação dos alunos, quais características do PBL acrescentam mais a seu conhecimento e crescimento profissional. Para as sete características destacadas, os alunos definiram uma hierarquia de importância numerando-as de 1 a 7 , que representam mais e menos importante respectivamente. Observa-se que a opção mais vezes citada como a mais importante foi "Desenvolvimento de Competência de Solução de Problemas", oito vezes. Outras que receberam destaques nesta opção foram "A Pesquisa Proporciona Autoaprendizagem" e "Estimula a Busca de Conhecimento", que também obtiveram quantidade em destaque na $2^{\mathrm{a}}$ posição da hierarquia. As opções menos bem posicionadas na maior parte dos questionários respondidos foram "Lidar com Problemas de Relacionamento" e "Instiga papéis diferentes no grupo". Tal resultado pode ser explicado pelo fato de os grupos terem sido compostos por poucos alunos, no máximo três, dificultando a ocorrência de papéis, como de liderança, ou até mesmo o surgimento de problemas maiores de relacionamento entre os integrantes do grupo. Contudo, a partir de uma média aritmética das posições escolhidas por cada questionário de cada item, observou-se que a variação da média entre o melhor e o pior item foi de 3,3 e 5,1, para as opções "A Pesquisa Proporciona Autoaprendizagem" e "Lidar com Problemas de Relacionamento", respectivamente.

Por último, duas perguntas respondidas pelos alunos avaliam o comportamento do professor em apresentação e dinamismo do conteúdo da disciplina, em apoio às atividades dos alunos, e o método de avaliação utilizado para julgar os trabalhos realizados pelos grupos. Os gráficos da Figura 5 mostram esses resultados. 


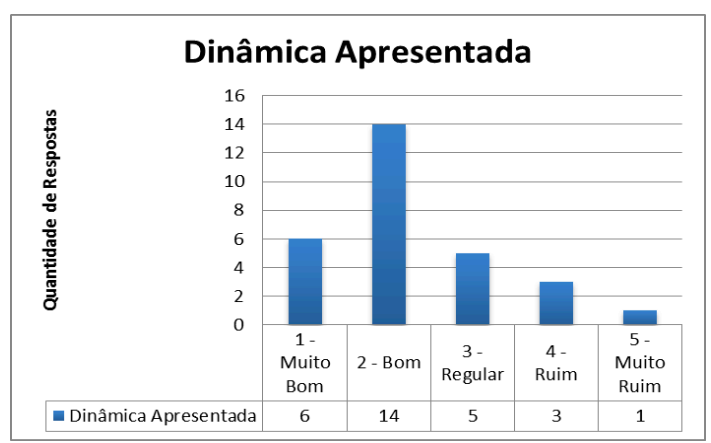

Figura 5 - Avaliação da dinâmica apresentada

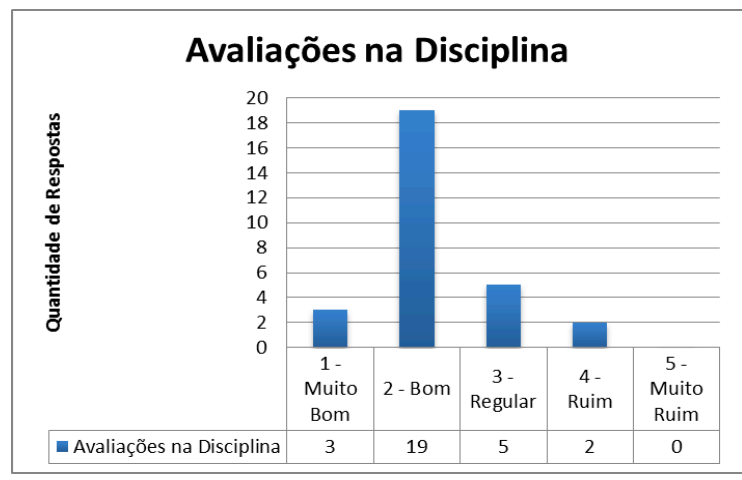

Figura 6 - Avaliação da forma de avaliação na disciplina IHC

O primeiro gráfico mostra que $68,9 \%$ dos alunos avaliaram positivamente as dinâmicas de apresentação de aulas, o que indica boa aceitação do método utilizado para expor o conteúdo necessário. Resultado ainda melhor foi obtido nos critérios de avaliação utilizados pelo professor da disciplina de IHC para avaliar os trabalhos realizados pelos alunos nos diferentes pontos de controle existentes ao longo da disciplina, resultando em 78,6\% de respostas positivas. Tais resultados são importantes, pois refletem o exercício do papel de professor como tutor dos trabalhos, que direciona a disciplina na apresentação dos conteúdos mais importantes para resolver problemas, assim como aplica critérios de avaliação adequados para aferir se o conhecimento esperado foi, de fato, absorvido pelos alunos.

\section{Considerações Finais}

O presente estudo buscou contribuir com os estudos sobre a utilização da abordagem de aprendizagem baseada em problemas na busca de validar a experiência de sua utilização no contexto da disciplina Interação Humano-Computador. A abordagem, que foi utilizada durante três semestre no contexto da disciplina IHC do curso Engenharia de Software da Faculdade UnB Gama, foi bem recebida, em média, pelos alunos.

Todos os resultados de perguntas com repostas objetivas - positivas e negativas - demonstraram a satisfação da maioria dos alunos com o método PBL para a disciplina de IHC, da forma como foi trabalhado naquele contexto. O questionamento que teve o maior equilíbrio entre respostas positivas e negativas foi a respeito da escolha dos problemas que seriam trabalhados. Cumpre salientar que, embora em todos os semestres tenha sido proposto um tema central pelo professor, como, por exemplo, aplicações para serem usadas no esporte, os alunos eram os responsáveis por explorar problemas específicos e trabalhar em possíveis soluções para atacar os problemas 
envolvidos. É provável que um maior envolvimento do professor na proposição dos problemas e na escolha de soluções possa melhorar a motivação dos alunos, uma vez que ele pode transpor sua experiência e conhecimento na escolha de temas mais favoráveis para bem trabalhar os conceitos da disciplina. Evidentemente, o grau de maturidade de adoção do PBL em IHC aumentou a cada semestre, possibilitando que o professor modelasse os problemas que seriam trabalhados e direcionasse a adoção e execução das soluções e pesquisas feitas e o método de avaliá-las.

Este estudo avaliou de maneira qualitativa e quantitativa algumas características do PBL. Buscou-se essencialmente enfatizar quais características os alunos achavam mais interessantes no uso da abordagem baseada em problemas com base numa seleção hierárquica de importância e não necessariamente explorar cada uma delas de maneira detalhada. Destacam-se, portanto, as características "A Pesquisa Proporciona Autoaprendizagem" como a característica do método que mais agrada aos estudantes e "Lidar com Problemas de Relacionamento" como a menos bem avaliada. O assunto merece estudos futuros para explorar características do PBL que mais contribuem para a formação do aluno, a construção de conhecimentos específicos, a capacidade de resolver problemas e outras, para que os professores possam melhor explorar as potencialidades da metodologia em diferentes contextos.

Portanto, neste estudo conclui-se que a adoção do PBL no contexto apresentado mostou-se excelente opção para melhorar o processo de ensino e aprendizagem, e que outras experiências, não só no ensino de IHC, mas em outras disciplinas da Engenharia de Software como um todo, devem ser encorajadas como contribuição para melhorar a utilização da abordagem baseada em problemas.

\section{Referências Bibliográficas}

BOSCARIOLI, C. (2011). O Ensino de IHC por Meio de Aprendizagem Baseada em Problemas: Um Relato de Experiência. WEIHC - Workshop sobre Ensino de IHC. Porto de Galinha, PE, 2011.

CERUTTI, D. M. L. Ensino de IHC: Desconstruindo interfaces em sala de aula. WEIHC - Workshop sobre Ensino de IHC. Belo Horizonte, MG, 2010.

GREENING, T.; KAY, J.; KINGSTON, J. H. Trialling a Problem-Based Learning Approach to First Year Computer Science. ACSE - Australian Conference on Computer Science Education. Melbourne, Victoria, 1997.

JUNIOR, P. T. A. Papéis do Docente em IHC: do conhecimento ao mercado. Ihc 2010 IX Simpósio sobre Fatores Humanos em Sistemas Computacionais. Belo Horizonte, MG. Anais Extendidos do IX Simpósio de Fatores Humanos em Sistemas Computacionais (Volume II). Porto Alegre - RS: Sociedade Brasileira de Computação SBC, 2010. v. 01.p. 79-82.

LINGE, N.; PARSONS, D. Problem-Based Learning as an Effective Tool for Teaching Computer Network Design. IEEE Transaction On Education, v 49, n.1, 2006.

NORDAHL, R.; SERAFIN, S. Using Problem Based Learning to Support Transdisciplinary in an HCI Education. Proceedings of Hcied (HCI In Education) Conference. Association for Computing Machinery, 2008. p. 94.

PEREIRA, C. F.; AFONSO, R. A.; SANTOS, M.J.; ARAÚJO, C. A. L.; NOGUEIRA, M. (2007). XIV SIMPEP - Simpósio de Engenharia de Produção. Bauru, SP. 
RIBEIRO, L.R.C.; ESCRIVÃO FILHO, E.; MIZUKAMI, M. G. N. Uma Experiencia com a PBL no Ensino de Engenharia Sob a Ótica dos Alunos. Revista de Ensino em Engenharia, $v 23, n .1, p .63-71,2004$.

RODRIGUES, E. A.; ARAÚJO, A. M. P. (2006). O Ensino de Contabilidade: Aplicação do Método PBL nas Disciplinas de Contabilidade em Uma Instituição de Ensino Superior Particular. VI Congresso USP Controladoria e Contabilidade. São Paulo, SP.

SALES, ANDRÉ BARROS DE ; COELHO, L. D. N. ; SALES, M. B. O Uso de Mapas Conceituais na Disciplina Interação Humano-Computador. Revista Extensio, (Florianópolis), v. 9, p. 66-72, 2012. 